Hai-Fang Li ${ }^{1}$

Jin-Ming Lin ${ }^{1}$

Rong-Guo Su${ }^{1}$

Zong Wei Cai ${ }^{2}$

Katsumi Uchiyama ${ }^{3}$

${ }^{1}$ Research Center for

Eco-Environmental Sciences,

Key Laboratory of Environmental

Chemistry and Ecotoxicology,

Chinese Academy of Sciences,

Beijing, China

${ }^{2}$ Department Chemistry,

Hong Kong Baptist University,

Kowloon Tong,

Hong Kong, China

${ }^{3}$ Department of Applied

Chemistry,

Faculty of Engineering,

Tokyo Metropolitan University,

Tokyo, Japan

\section{A polymeric master replication technology for mass fabrication of poly(dimethylsiloxane) microfluidic devices}

A protocol of producing multiple polymeric masters from an original glass master mold has been developed, which enables the production of multiple poly(dimethylsiloxane) (PDMS)-based microfluidic devices in a low-cost and efficient manner. Standard wetetching techniques were used to fabricate an original glass master with negative features, from which more than 50 polymethylmethacrylate (PMMA) positive replica masters were rapidly created using the thermal printing technique. The time to replicate each PMMA master was as short as $20 \mathrm{~min}$. The PMMA replica masters have excellent structural features and could be used to cast PDMS devices for many times. An integration geometry designed for laser-induced fluorescence (LIF) detection, which contains normal deep microfluidic channels and a much deeper optical fiber channel, was successfully transferred into PDMS devices. The positive relief on seven PMMA replica masters is replicated with regard to the negative original glass master, with a depth average variation of $0.89 \%$ for $26 \mu \mathrm{m}$ deep microfluidic channels and $1.16 \%$ for the $90 \mu \mathrm{m}$ deep fiber channel. The imprinted positive relief in PMMA from master-to-master is reproducible with relative standard deviations (RSDs) of $1.06 \%$ for the maximum width and $0.46 \%$ for depth in terms of the separation channel. The PDMS devices fabricated from the PMMA replica masters were characterized and applied to the separation of a fluorescein isothiocyanate (FITC)-labeled epinephrine sample.

Keywords: Microfluidic device / Miniaturization / Poly(dimethylsiloxane) / Thermal printing / Wet-etch

\section{Introduction}

In the past decade, the development of microscale (chipbased) separation devices has experienced an explosive growth in both industrial and academic fields worldwide [1]. Most of the devices reported to date have been fabricated in glass or silicon [2-6]. However, the current cost of producing high-performance glass or silicon-based microfluidic devices prohibits their utilization as disposable devices. Polymer-based microchips, which are less expensive and easier to manipulate than glass and silicon devices, have begun to show their vast potential in the field of microscale analysis. The primary advantage of polymer substrates lies in the ability for mass production

Correspondence: Prof. Dr. Jin-Ming Lin, Research Center for Eco-Environmental Sciences, Key Laboratory of Environmental Chemistry and Ecotoxicology, Chinese Academy of Sciences, P.O. Box 2871, Beijing, 100085, China

E-mail: jmlin@mail.rcees.ac.cn

Fax: +86-10-62841953

Abbreviations: BOE, buffered oxide etchant; CAD, computeraided design; PB, hexadimethrine bromide (Polybrene); PDMS, poly(dimethylsiloxane); PMMA, polymethylmethacrylate; SEM, scanning electron microscope at low costs based on replication (casting, embossing, imprinting, or injection molding) techniques for the possible disposable use [7]. Several important polymers including poly(dimethylsiloxane) (PDMS) [8-11], polymethylmethacrylate (PMMA) [12-15], polycarbonate [16, $17]$, and polystyrene $[18,19]$ are now increasingly used to fabricate microfluidic systems. In particular, PDMS is a soft polymer that is being actively developed for miniaturized bioassays due to its desirable features including easy replica molding, good optical transparency (down to $230 \mathrm{~nm}$ ), no toxicity to proteins and cells, as well as easy irreversibly or reversibly sealing to itself and other materials [9, 20-23].

Currently, the common techniques for the fabrication of PDMS devices are mainly focused on the high resolution of the microstructures rather than on high throughput. PDMS devices were fabricated by casting replicas against a positively patterned rigid mold or master [10]. The primary masters used for casting PDMS were etched silicon or glass with positive reliefs [8]. However, the disadvantages of high costs and time-consuming procedure have restrained their application, especially for industrial 
production. To accelerate and simplify the production process, several rapid prototyping approaches for the fabrication of PDMS-based microfluidic devices have been recently developed. Whitesides et al. [9] used a positive photoresist master adhered to a silicon wafer to cast PDMS devices. A patterned high-resolution transparency mask instead of a chrome mask was employed to rapidly create the photoresist master. With this technique, PDMS microfluidic devices $>20 \mu \mathrm{m}$ can be produced at low costs within $24 \mathrm{~h}$. Subsequently, Whitesides et al. [24] developed another prototyping approach for creating three-dimensional masters for molding PDMS devices using the solid-object printing technique. The design of the three-dimensional microstructure was made by using a computer-aided design (CAD) program. Then the CAD file was read by a commercial solid-object printer which directly fabricated a master in a thermoplastic material without use of a mask. Compared with conventional photolithography, the solid-object printer reduced the number of steps in fabrication of masters. However, the generated structures not only were easily breakable, but also had poorer resolution (features of $>250 \mu \mathrm{m}$ ) and larger surface roughness $(\sim 8 \mu \mathrm{m})$. Another important rapid prototyping of masters for PDMS devices was explored based on liquid-phase photopolymerization techniques [25]. Masters were fabricated by dispensing a liquid prepolymer mixture on a silicon substrate and exposing it to UV light through a photomask. The liquid masked from the light remains unpolymerized and can be washed away, while the liquid exposed to the light becomes polymerized and cured. While the photoresist masters can be rapidly produced via photolithography and are more flexible, the insufficient hardness and deformation generally led to geometries collapsing and releasing from backstops. For a commercial production, this kind of masters have too limited lifetime and production runs.

Recently, some metal materials have been used as master mold substrates. For example, Sudarsan and Ugaz [26] used conventional printed circuit technology to fabricate master molds in photosensitized copper clad circuit board substrates. This process could yield features more than $50 \mu \mathrm{m}$ in width and $15-120 \mu \mathrm{m}$ in height through selection of an appropriate copper layer thickness. In particular, the surface roughness of the printed circuit board's insulating substrate layer may prevent sufficient adhesion of the PDMS replica to a flat PDMS or other materials.

While each of these prototyping processes described above have been successfully used for the fabrication of PDMS microfluidic systems, they are more suitable for the production of limited numbers of microdevices than for a commercial production. Since the speed of molding PDMS from a master is 1-2 h per replica, large numbers of masters are needed for realizing high throughput. For fabrication of large numbers of masters, the techniques of pattering dense photoresist materials or directly etching features into silicon substrates are inefficient and cleanroom facilities are always required. Moreover, to achieve good reproducibility of these individually fabricated masters, steps such as spin-coating photoresist, UV exposal, and etching must be carefully controlled.

Because master fabrication is a time-consuming step of massive production PDMS devices, master replication techniques were explored to bypass the typical one-byone master fabrication process. McCormick et al. [12] used an electroform method to simultaneously replicate multiple nickel electroform "daughter" injection molds from a nickel "mother" template that was prepared from a wet-etched negative silicon template. Another approach involves cutting an aluminum mask with a high-precision mill, and then creating an acrylic master from the aluminum mask [27]. The master replication process of heating the acrylic at above $200^{\circ} \mathrm{C}$ for $2 \mathrm{~h}$ in an oven to transform its surface into viscous liquid and sink into the negative features of the aluminum mask could reproduce features from 7 to $20 \mu \mathrm{m}$ in height. The replication technique was not able to replicate features of more than $20 \mu \mathrm{m}$ [27], and that was unsuitable for some integration systems with deeper functions for particular analysis.

In this report, we describe a simpler and faster master replication technique, which allows many reproducible PDMS devices to be cast simultaneously at low costs. Commercially available PMMA sheets were used as replica master material since they can be easily structured against a silicon template by thermal imprinting [28]. In addition, the hard and slick physical property of PMMA at $<100^{\circ} \mathrm{C}$ makes it suitable for low-temperature casting of PDMS. In this strategy, an original glass negative master was firstly fabricated using standard wet-etching techniques, from which multiple PMMA positive replica masters were created. These PMMA replica masters can be repeatedly used for PDMS-based device casting with minimal degradation. Therefore, this master replication technique specialized in PDMS devices mass production contrasted with other reported master fabrication techniques. The remarkable advantage of this technique originating from a glass negative master is that a more flexible and rugged geometry can be easily obtained compared to the direct etching of a positive structure in glass. This is significant because different sizes of functional components and interconnects are generally required for realizing the integration detection purpose. In our experiments, a much wider and deeper optical fiber channel 
than microfluidic channels was integrated in the device for constructing an integration LIF detection system. The reproducibility and validity of the fabrication procedure was characterized and the PDMS devices made from PMMA replica masters were applied for separation of a fluorescein isothiocyanate (FITC)-labeled epinephrine sample.

\section{Materials and methods}

\subsection{Chemicals and solutions}

All reagents used in our experiments were of analytical reagent grade. FITC, hexadimethrine bromide (Polybrene, $\mathrm{PB}$ ), and epinephrine were purchased from Sigma (St. Louis, MO, USA). Two differently concentrated $\mathrm{Na}_{2} \mathrm{~B}_{4} \mathrm{O}_{7} /$ $\mathrm{HCl}, \mathrm{pH} 9.0$, buffer solutions ( 10 and $20 \mathrm{~mm}$ ) were used for electroosmotic flow (EOF) studies. A $5 \mathrm{~mm}$ stock solution of epinephrine was prepared in $10 \mathrm{~mm} \mathrm{Na} \mathrm{B}_{4} \mathrm{O}_{7} / \mathrm{HCl}, \mathrm{pH}$ 9.0, buffer. $40 \mathrm{~mm}$ FITC labeling reagent was prepared in acetone with $1 \% \mathrm{v} / \mathrm{v}$ pyridine added and $20 \mu \mathrm{M}$ aqueous solution of FITC was prepared in $\mathrm{Na}_{2} \mathrm{~B}_{4} \mathrm{O}_{7} / \mathrm{HCl}$ buffer for testing the durability of the PB layer coated onto the channels walls. All solutions were filtered through $0.45 \mu \mathrm{m}$ membrane filters prior to use.

\subsection{Fabrication}

The sequence of the fabrication process of the PDMS chips is schematically depicted in Fig. 1, which can be broadly divided into three steps: (i) wet-etching of an original glass negative mold (Fig. 1A); (ii) thermal-imprinting of PMMA positive replica masters against the glass mold (Fig. 1B); and (iii) replica molding of PDMS against PMMA replica masters (Fig. 1C). The glass negative master (Fig. 1A) was fabricated by using conventional photolithographic pattern transfer and wet chemical etching techniques. A $1.4 \mathrm{~mm}$ thick soda lime glass $(6.3 \mathrm{~cm} \times 6.3 \mathrm{~cm})$ precoated with $145 \mathrm{~nm} \mathrm{Cr}$ layer and $570 \mathrm{~nm}$ positive AZ1518 photoresist (Shaoguang Microelectronics, Changsha, China) was used as the substrate for the glass negative master. A design of a microfluidic device for LIF detection was created in a CAD program and then imprinted onto a transparency with a high-resolution commercial image setter [9]. As shown in Fig. 2, the design includes a cross microchannel and two wider symmetry optical fiber channels. The transparency served as a mask to transfer the channel structure onto the glass substrate following UV exposure. The negative structure was etched into the glass substrate in a stirred $0.3 \mathrm{M}$ buffered oxide etchant (BOE, $\mathrm{HF}: \mathrm{NH}_{4} \mathrm{~F}=1: 1$ ) at room temperature. The channel size was checked under
A Glass mold

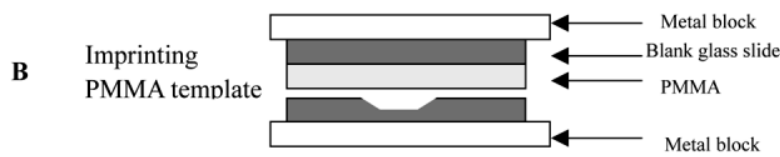

PMMA template $\square$

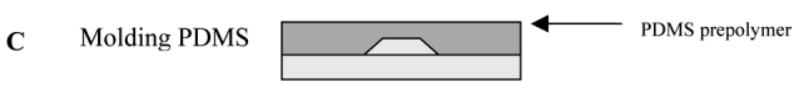

PDMS replica

Figure 1. Fabrication of PDMS microfluidic systems: (A) wet-etched original glass negative master; (B) imprinting PMMA replica master against the glass master and obtained PMMA replica master with positive surface relief; (C) casting PDMS against the PMMA replica master and peeling the cured PDMS replica off from the PMMA replica master.

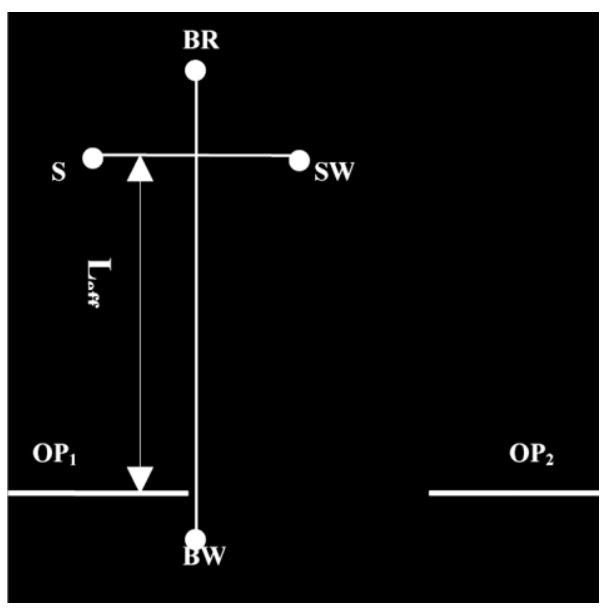

Figure 2. Design of the photomask used to fabricate the original glass negative master. The channel lengths from the cross to the buffer reservoir (B), sample reservoir (S), sample waste reservoir (SW), and buffer waste reservoir (BW) were 1, 1, 1, and $4 \mathrm{~cm}$, respectively; effective separation length $\left(L_{\text {eff }}\right), 3.8 \mathrm{~cm}$. The optical fiber channels were denoted as $\mathrm{OP}_{1}$ and $\mathrm{OP}_{2}$, which were $300 \mu \mathrm{m}$ away from the separation channel.

a microscope every 15-30 min. Once the dimension of the microfluidic channel reached the expected size, they were shielded by waterproof adhesive tape. The glass substrate was then transferred into $1.0 \mathrm{~m} \mathrm{BOE}$ to expand the fiber channels further until it fits an optical fiber (140 $\mu \mathrm{m}$ OD).

PMMA positive replica masters were replicated from the etched glass negative master with a thermal imprinting technique (Fig. 1B). The commercial $1.6 \mathrm{~mm}$ thick PMMA 


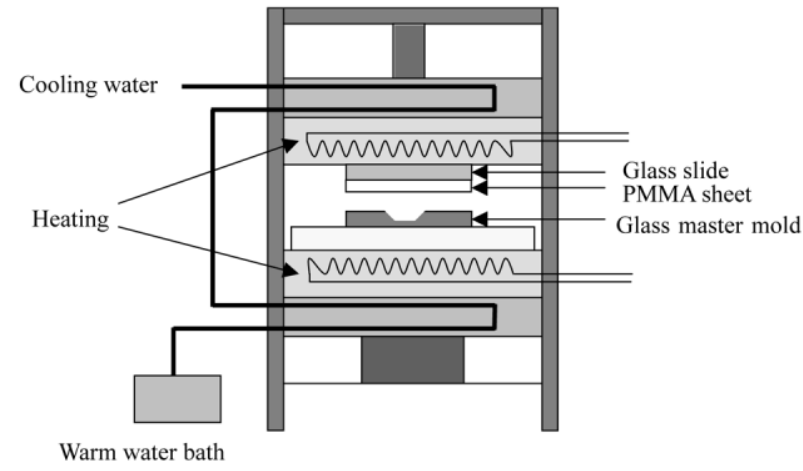

Figure 3. Diagram of a heat imprinting machine.

Plexiglas was cut into the same size to the glass negative master. The PMMA sheet was sandwiched between the glass master and a blank glass slide. All of their surfaces were cleaned prior to channel formation. The "sandwich" was placed between metal heating blocks of an imprinting machine (Fig. 3). The raised structure in PMMA was formed by heating the plastic at $115^{\circ} \mathrm{C}$ for 2 min with a pressure of $3.0 \mathrm{MPa}$. Then the "sandwich" was rapidly cooled down to $95^{\circ} \mathrm{C}$ by cooling water, which was collected into a tank serving as a warm bath. The voluntary demolding process was accomplished when the "sandwich" was removed from the imprinting machine into the $35-40^{\circ} \mathrm{C}$ bath. This imprinting procedure had been used to successfully pattern over 50 PMMA replica masters from the original glass master.

Replica molding of PDMS against the positive PMMA replica masters was easily accomplished by using a rapid prototyping method reported in the literature (Fig. 1C) [10]. The PMMA replica master was cleaned with deionized water and ethanol, and dried with nitrogen gas. A mixture of 10:1 of prepolymer and curing agent (Sylgard 184; Dow Corning, Midland, MI, USA) was stirred thoroughly and degassed in vacuum for $15 \mathrm{~min}$. Then the prepolymer mixture was poured onto a PMMA replica master and cured at $60^{\circ} \mathrm{C}$ for $1 \mathrm{~h}$. After peeling from the PMMA replica master, the PDMS replica was cut into two halves, with one containing the fluidic channels serving as the base plate and the other half serving as the cover plate. The access holes of $5 \mathrm{~mm}$ diameter at the ends of the fluidic channels on the base plate were drilled using a borer to serve as reservoirs. Irreversible sealing of PDMS was accomplished according to the previously published methods $[9,10]$. The cleaned plates were oxidized in a plasma discharge for $50 \mathrm{~s}$ and brought into conformal contact within $1 \mathrm{~min}$ after removal from the plasma. Prior to sealing, the complementary optical fiber channels in the two plates were specially aligned.

\subsection{Dynamic coating of fluidic channels and electrophoresis}

The sealed PDMS devices were dynamically coated with the cationic polymer of $\mathrm{PB}$ as reported in the literature with a small modification [29]. Briefly, the microfluidic channels were subsequently preconditioned with deionized water, $0.1 \mathrm{~m} \mathrm{NaOH}$, and deionized water, for $2 \mathrm{~min}$ each rinse. Then the channels were filled with $5 \%$ PB dissolved in water/methanol (1:1 v:v) for $2 \mathrm{~min}$, followed by a 15 min waiting period prior to use. The coated PDMS devices were applied to analyze the biochemical sample of epinephrine with LIF detection. Epinephrine was labelled with FITC at a dye-to-epinephrine ratio of 5:2 [30]. A solution of $10 \mathrm{~mm} \mathrm{Na} \mathrm{B}_{4} \mathrm{O}_{7} / \mathrm{HCl}(\mathrm{pH}$ 9.0) was served as running buffer for the capillary zone electrophoresis separation. The FITC-labeled epinephrine sample was diluted to $1 \times 10^{-5} \mathrm{M}$ with the $10 \mathrm{~mm}$ running buffer. Sample injection was achieved by grounding the sample reservoir (S) and applying a positive potential of $0.4 \mathrm{kV}$ at the sample waste reservoir (SW). Separation was performed by applying a high potential of $3.0 \mathrm{kV}$ at the buffer waste reservoir (BW) when the buffer reservoir (B) was grounded.

\subsection{LIF detection}

A simple laboratory-made integrated optical setup that was reported previously [31] was used for the fluorescence detection. A $473 \mathrm{~nm}$ blue diode-pumped laser (Beijing Viasho Technology, China) was used for excitation. The laser beam was focused by a plano-convex lens and coupled into a multimode optical fiber $(100 \mu \mathrm{m}$ core diameter and $140 \mu \mathrm{m}$ cladding diameter). The launch tip was inserted into the fiber channel of the PDMS device which was oriented perpendicularly to the separation microchannel. Fluorescence was directly detected by a photomultiplier tube after passing through a pair of holographic notch plus filter ( $476 \mathrm{~nm}$ ) and interference filter $(535 \mathrm{~nm})$, and a $400 \mu \mathrm{m}$ diameter pinhole. A CDY-800L high-voltage switching apparatus consisting of four $8 \mathrm{kV}$ high-voltage power supplies (Shandong Chemical Engineering Institute, China) was used. The detection point was $3.7 \mathrm{~cm}$ from the intersection point along the separation channel.

\section{Results and discussion}

\subsection{Creation of the original glass negative master}

The glass negative master was created by the combination of transparency photomask and a sequential etching procedure. The small-sized microfluidic channels were 
achieved by carefully etching in a well-stirred $0.3 \mathrm{M} \mathrm{BOE}$ solution for $1.5 \mathrm{~h}$. Then the optical fiber channels were widened at an accelerated rate in a $1.0 \mathrm{~m} \mathrm{BOE} \mathrm{solution} \mathrm{for}$ $5 \mathrm{~h}$. It was found that the etching of a negative structure in glass substrate was easier and faster than etching a positive glass mold. Under the same etching condition, the speed of etching a negative structure was 5 times faster than that of etching the same depth of a positive structure. Although the etching process took some time, employing an original glass negative mold to reproduce PMMA positive masters was advantageous: (i) the HFetched fluidic channel surface has a much lower roughness $(<100 \mathrm{~nm})$ due to the low removal rate of about $0.29 \mu \mathrm{m} / \mathrm{min}$; (ii) because all not-designed areas of glass can be protected and only the structure area was exposed during the etching process, more rugged and flexible negative features could be etched in glass than a positive relief. Thus, different heights and widths of the functional components could be integrated into the devices while keeping the surface adequately uniform; (iii) unlike all of the positive masters, the glass negative master could reproduce multiple PMMA replica masters. As the typical glass chip fabrication, we could rapidly and reliably create features in the glass substrate with widths of $>20 \mu \mathrm{m}$ which were limited by the resolution of the transparencies.

\subsection{Fabrication of the positive PMMA replica masters}

\subsubsection{Effect of imprinting conditions}

The imprinting PMMA step does not need special or complicated equipments. A commercially available inexpensive and simple heat imprinting machine (769YP-15A, Tianjin KEQI High and New Technology Co., China) was used. Imprinting temperature, pressure, and demolding temperature are important parameters that may influence the quality of the imprinting structure of PMMA. In our experiments, the PMMA could be well structured at $115^{\circ} \mathrm{C}$ that was chosen on the basis of its softening temperature $\left(105^{\circ} \mathrm{C}\right)$ [32]. If the pressing temperature was much lower or higher than the softening temperature, the resulting imprinted ridges could not fully represent the negative structure of the glass master due to the insufficient or excessive transformation of the PMMA surface. The demolding temperature directly related the obtained imprinting structure of PMMA. The optimal temperature for demolding PMMA from the glass negative master was found to be $94-98^{\circ} \mathrm{C}$. Larisa et al. [28] have reported that the positive sub- $\mu \mathrm{m}$ features of silicon templates could be successfully reproduced to PMMA by removing the PMMA from the silicon template while still warm from the press; otherwise, the negative imprinting channels were jagged with a stairstep-like appearance caused by their different thermal expansion properties. It should be mentioned that if the PMMA was mechanically removed from the glass master by force at a high temperature, the formed features in PMMA would be easily breached. Therefore, a voluntary demolding method was created based on different thermal expansion properties between PMMA and glass master mold, i.e., the power of departing PMMA away from the glass master came from their different contractile forces caused by the prompt temperature decline. As observed in the experiments, the PMMA detached from the glass mold as soon as the hot "sandwich" was submerged in a warm bath at $30-40^{\circ} \mathrm{C}$. After reproducing more than 50 pieces of PMMA replica masters, the structural feature of the glass negative master was still observed under the optical microscope. It was found that there was no apparent defacement compared with the fresh glass master. The whole cycle for each PMMA replica master, including assembling, imprinting, and demolding, was as short as $20 \mathrm{~min}$. Although the time for wet-etching a glass negative master was longer compared to the emerging rapid prototyping techniques, it could be compensated if many PMMA replica masters were reproduced.

Besides the temperature effects, pressure is another critical factor in the imprinting process. A moderate and uniform press is required to act on the surface of the glass master mold. The pressure of $3.0 \mathrm{MPa}$ was adequate to impel the softened plastic filled in the negative channel of the glass master. Uneven or too high pressure not only affected the channel profile in PMMA replica masters but also induced the fragmentation of the glass master.

\subsubsection{Characteristics of the imprinted PMMA replica masters}

As shown in Figs. 4A and B, optical images of intersections of the glass negative master and a PMMA replica master obtained using an imaging system (Motic images, Motic China Group) demonstrate that the positive relief of the PMMA replica master correlates quite well with the negative structure of the glass master. Moreover, by the thermal imprinting process PMMA accurately reproduces the smooth surface of the glass master. The resolution of structural features of glass master and PMMA replica were characterized by profile scan measurements with a profilemeter (Mahr, Concept, Perthometer PGK 120). The isotropic nature of the glass master etching process yielded trapezoidal channels, whereas the thermal imprinting process made an inverse-raised three-dimensional image on PMMA. For the glass master, the separation channel has an average dimension of $26.3 \mu \mathrm{m}$ depth, $134.1 \mu \mathrm{m}$ 


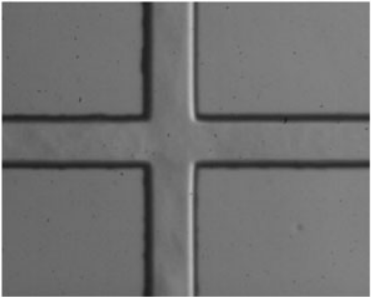

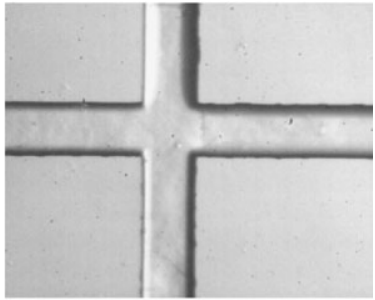

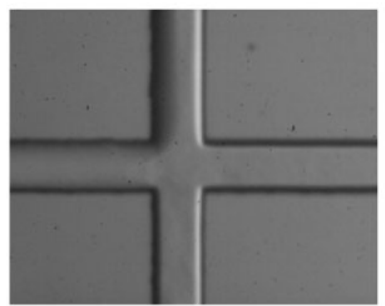

Figure 4. Microscopic images of channels. (A) Intersection of the negative relief channel on the glass mold; (B) intersection of the positive relief channel on the PMMA replica master; $(C)$ intersection of the channel on the PDMS replica.

maximum width on the top, and $89.8 \mu \mathrm{m}$ minimum width on the bottom. The optical fiber channels have an average dimension of $90.2 \mu \mathrm{m}$ depth, $400.6 \mu \mathrm{m}$ maximum width on the top, and $240.3 \mu \mathrm{m}$ minimum width on the bottom. For the 50 pieces of PMMA replica masters, the separation channels have an averaged dimension of $26.2 \mu \mathrm{m}$ height, $89.2 \mu \mathrm{m}$ minimum width at the top, and $135.8 \mu \mathrm{m}$ maximum width on the bottom. The optical fiber ridges have averaged depth of $89.1 \mu \mathrm{m}$, minimum width of $238.2 \mu \mathrm{m}$ on the bottom, and maximum width of $396.4 \mu \mathrm{m}$ on the top.

To assess the reproducibility of the parallel fabrication of PMMA replica masters, separation channel dimensions of seven PMMA replica masters were measured at corresponding locations as shown in Table 1. No. 1 and No. 7 were the first and the 50th PMMA obtained, respectively, and the other five replicas were randomly chosen. Results in Table 1 indicate that the variation of the PMMA replica masters from the glass mold in terms of the separation channel was small, with relative standard deviations (RSDs) of $0.89 \%$ for the depth and $1.14 \%$ for the maximum width. The reproducibility of PMMA master-tomaster in terms of separation channel dimensions was also very well, with low RSDs of $0.46 \%$ for the depth and $1.06 \%$ for the maximum width. The low RSDs indicate the good reproducibility of the imprinting process and durability of the glass mold.
Table 1. Dimensions of the imprinted PMMA replica master separation channels

\begin{tabular}{lll}
\hline PMMA master No. & Width $_{\max }(\mu \mathrm{m})^{\mathrm{a})}$ & Depth $(\mu \mathrm{m})$ \\
\hline 1 & 135.1 & 26.3 \\
2 & 133.8 & 26.2 \\
3 & 133.4 & 26.0 \\
4 & 136.5 & 26.0 \\
5 & 135.3 & 26.2 \\
6 & 132.4 & 26.1 \\
7 & 135.6 & 26.0 \\
\hline
\end{tabular}

a) Width max $_{\text {is }}$ the maximum width of the separation channel on the PMMA replica masters.

Table 2. Dimensions of four points of microstructures of the PMMA replica master and that of the glass master mold

\begin{tabular}{lll}
\hline $\begin{array}{l}\text { Glass master mold } \\
\text { (PMMA master) })^{a}\end{array}$ & Depth $(\mu \mathrm{m})$ & Width $_{\max }(\mu \mathrm{m})$ \\
\hline $\mathrm{A}_{\mathrm{g}}\left(\mathrm{A}_{\mathrm{p}}\right)$ & $26.1(26.0)$ & $128.4(131.4)$ \\
$\mathrm{B}_{\mathrm{g}}\left(\mathrm{B}_{\mathrm{p}}\right)$ & $26.3(26.3)$ & $134.1(135.1)$ \\
$\mathrm{C}_{\mathrm{g}}\left(\mathrm{C}_{\mathrm{p}}\right)$ & $25.8(25.6)$ & $132.4(133.3)$ \\
$\mathrm{D}_{\mathrm{g}}\left(\mathrm{D}_{\mathrm{p}}\right)$ & $25.7(26.1)$ & $124.3(125.5)$ \\
\hline
\end{tabular}

a) $A_{g}, B_{g}, C_{g}, D_{g}$ delineate the points of microstructures of the negative glass master; $A_{p}, B_{p}, C_{p}, D_{p}$ delineate the corresponding points of the replicated microstructures of the PMMA master.

The deviation of the channel profile between the individual PMMA replica master and the glass master was investigated. Four different locations along the microfluidic channels, i.e., A and B points on the separation channel as well as $C$ and $D$ points on the sample injection channel, were measured. The results listed in Table 2 show that the channel variations are small or insignificant for the entire PMMA replica master.

\subsection{Production of PDMS devices from the PMMA replica masters}

During the process of replica casting PDMS devices from the imprinted PMMA replica master, PDMS did not adhere to the masters and thus could be easily demolded without using any releasing agents. Figure $4 \mathrm{C}$ shows the obtained PDMS channel profile at the cross intersection, which is identical as that of the PMMA replica master shown in Fig. 4B. Figure 5 demonstrates the results obtained from scanning electron microscope (SEM) image measurement (JSM-35C, JEOL), indicating that the intersection of the PDMS replica is highly comparable to 


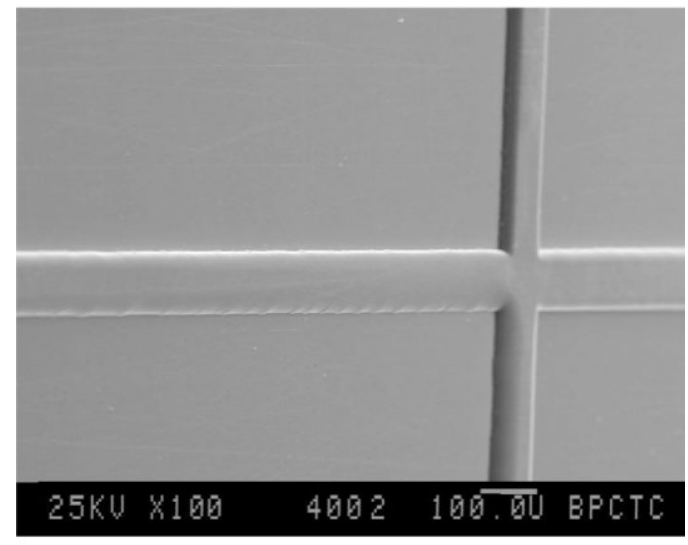

A

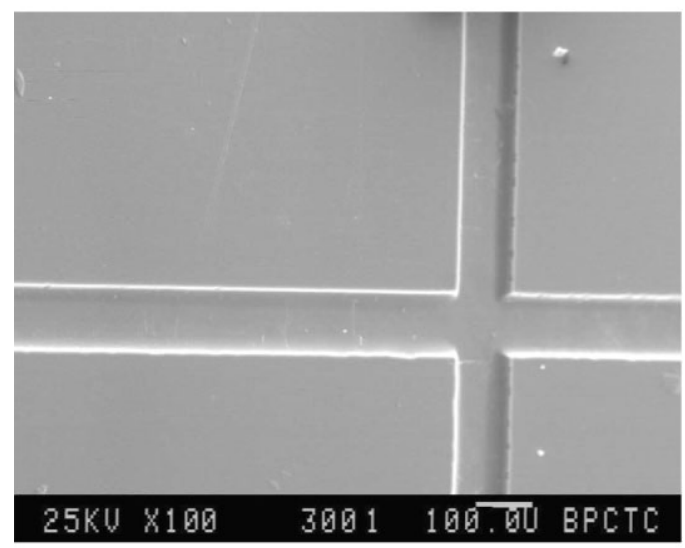

B

Figure 5. Scanning electron micrographs of $(A)$ the injection region of the PMMA replica master and $(B)$ the injection region of the unsealed PDMS substrate. The small defects visible on the walls and at the bottom of the channel may be due to artifacts from the gold sputtering process.

the corresponding PMMA replica master. As shown in the SEM images, the $90^{\circ}$ angle between the separation and injection channels was perfectly transferred from the PMMA replica master to the PDMS replica. The photo of a sealed PDMS chip fabricated from the PMMA replica master is shown in Fig. 6. The smooth area surrounding the microfluidic channels on the PMMA-fabricated PDMS replica allows its easy and uniform bonding with the blank PDMS replica.

The attenuated total reflection infrared (ATR-IR) spectra (Spectrum GX; Perkin Elmer, Norwalk, CT, USA) of the PDMS molding from the PMMA replica master and the PDMS molding from a microscope slide were compared. The results showed that the ATR-IR spectra of the both PDMSs had identical absorption bands. The PMMA replica master was not affected with the PDMS prepolymer and the curing agent. With the temperature range of $60-80^{\circ} \mathrm{C}$ that is commonly used for PDMS curing, the PMMA replica masters are robust and can be repeatedly used. No significant deformation of PMMA replica masters was observed after casting a series of PDMS. In addition, the dimensions of ten PDMS replicas cast from the No. 1 PMMA replica master in Table 1 were examined. The results indicated that all structures were similar to the positive structure of the PMMA replica master, with RSDs of $0.53 \%$ in depths and $1.25 \%$ in maximum widths.

\subsection{Microfluidic device}

We have fabricated a number of PDMS microfluidic devices to investigate the feasibility of this fabrication technique strategy and the reproducibility of device-to-device. A chip shown in Fig. 6 illustrates an obtained PDMS device that has a typical trapezoidal channel cross-section of $111 \times 26 \mu \mathrm{m}$. The unitary optical fiber channel has an averaged width of $317 \mu \mathrm{m}$ and depth of $178 \mu \mathrm{m}$. The total length of the fiber channel was $1.2 \mathrm{~cm}$ and the distance between the separation channel and the end of the optical fiber channel was only $300 \mu \mathrm{m}$.

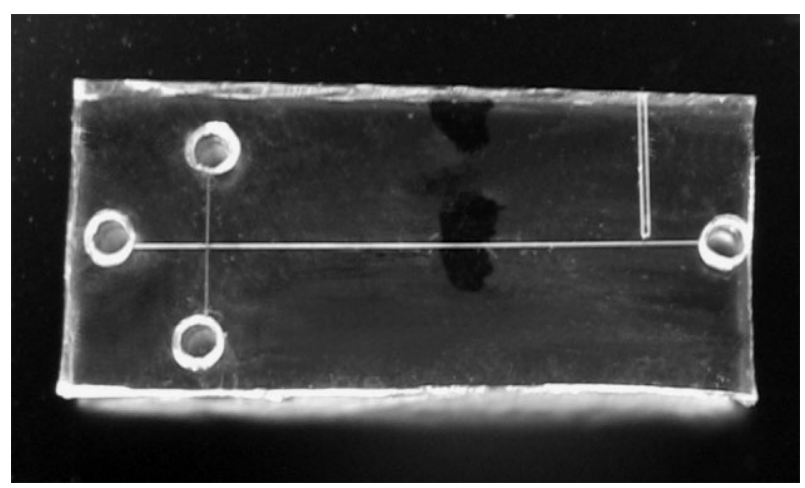

Figure 6. Photograph of a sealed PDMS chip fabricated from a PMMA replica master.

\subsection{Electroosmotic flow}

In CE separation, EOF is a wall-generated phenomenon that typically dominates the linear flow velocity of both the run buffer and the analytes being separated. One significant problem with unmodified PDMS microfluidic devices is the poorly defined EOF. Morra et al. [33] have reported that the contact angle of water on oxidized PDMS left open to the atmosphere changed from $30^{\circ}$ to $79^{\circ}$ in $15 \mathrm{~min}$ and the contact angle $93^{\circ}$ almost back to the value of native PDMS after $45 \mathrm{~min}$. Liu et al. [29] have found that 


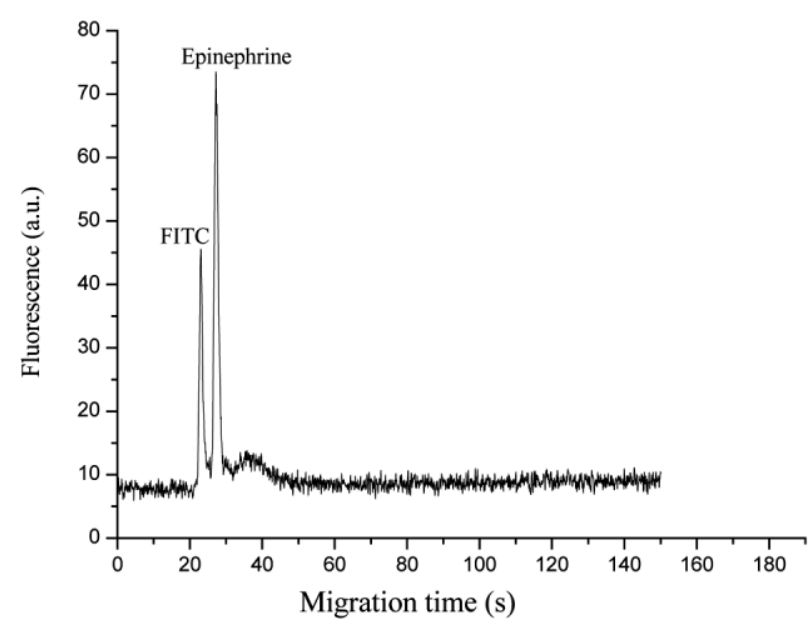

Figure 7. Electropherogram of the separation of FITClabeled epinephrine.

the PB dynamically coated PDMS/glass chips exhibited stable reversible direction EOF. In our experiment, five PDMS devices prepared from five corresponding PMMA replica masters, respectively, were selected for the EOF investigation. The EOF of these uncoated and PB-coated PDMS devices was measured by using the reported current monitoring method [29, 34, 35]. Two differently concentrated (10 and $20 \mathrm{~mm}$ ) $\mathrm{Na}_{2} \mathrm{~B}_{4} \mathrm{O}_{7} / \mathrm{HCl}(\mathrm{pH}$ 9.0) buffer solutions were used. Briefly, the channels and reservoirs were first filled with the $10 \mathrm{~mm}$ buffer and a constant current was obtained. Then the $10 \mathrm{~mm}$ buffer in all the reservoirs except for the waste reservoir was replaced with $20 \mathrm{~mm}$ buffer. Under the electrical field action, the EOF drove the $20 \mathrm{~mm}$ buffer to gradually fill the whole separation channel. The time needed to obtain another constant current is considered to be the EOF rate migration time. The electroosmotic velocity $\mu_{\mathrm{eo}}$ was calculated according to $\mu_{\mathrm{eo}}=(L / t) E^{-1}$ where $L$ is the length of the whole separation channels $(4.7 \mathrm{~cm}), t$ is the time for the diluted solution to be completely displaced by the concentrated solution in the separation channels, and $E$ is the added electric field strength. For the uncoated and PB-coated PDMS device determination, the positive and negative potentials are employed, respectively. Measurements were made at a field strength of $\pm 600 \mathrm{~V} / \mathrm{cm}$. The average electroosmotic mobilities were obtained from three consecutive measurements. The uncoated oxidized PDMS channel supported an EOF towards the cathode. The average value of EOF of the five PDMS devices was $3.5 \times$ $10^{-4} \mathrm{~cm}^{2} \mathrm{~N} \cdot \mathrm{s}$, with a device-to-device variation of $7.8 \%$. The EOF of the PDMS devices after PB-coating was reversed towards the anode and the average value was $-7.1 \times 10^{-5} \mathrm{~cm}^{2} / \mathrm{N} \cdot \mathrm{s}$, with a device-to-device variation of $6.2 \%$.

\subsection{Electrophoresis}

The robustness of the PB layer coated onto the channel walls was tested by a series of sequential injections of $20 \mu \mathrm{M}$ FITC. The results showed that the fluorescence signal intensity varied less than $10 \%$ within 25 runs. Under the negative potential, the cationic polymer PBcoated PDMS device successfully separated the anionic sample of FITC-labeled epinephrine with LIF detection. The resulting electropherogram is shown in Fig. 7 . There was no evidence of adsorption of the analytes to the surface of the channel walls since the peaks of the analytes are sharp and symmetric and the baselines are low and stable throughout repeated separations.

\section{Concluding remarks}

We have developed a polymeric master replication technique to realize the massive production of PDMS-based disposable devices in a rapid and low-cost manner. The fabrication steps of an original glass negative master including designing and imprinting a high-resolution transparency mask, UV exposing, and wet-etching can be completed in several hours. The subsequent step of reproducing multiple PMMA positive replica masters from the original glass master using the thermal imprinting technique takes only 20 min per PMMA replica. More than 50 pieces of PMMA replica masters were successfully produced. With these PMMA replica masters, if desired, disposable PDMS devices can be produced in a highthroughput manner. The reproducibility of the fabrication procedure was excellent and the validity was small. Optical and SEM images showed that the structure dimensions of PDMS replica matched well with those of the PMMA replica masters. Under the same conditions it was found that the EOF values of the PDMS microfluidic devices replicated from the PMMA replica masters exhibited good reproducibility, indicating that these PDMS devices had the similar separation capabilities towards the same analytes. This device-to-device reproducibility is significant for academic research as well as industrial production since many research works need multiparallel or sequential analyses. The master replication technique may also be important for the fabrication of large numbers of gel or packaging electrophoresis chips.

Compared to the conventional fabrication techniques of PDMS devices, our approach represents certain advantages: (i) the strategy of creating an original structure as negative relief in glass facilitates more flexible integration geometries and smoother appearance for devices to be obtained. The minimum width of the fabrication channel depends on the resolution of the printed transparency 
mask ( $>20 \mu \mathrm{m})$. (ii) Once an original glass master is fabricated, multiple PMMA replica masters can be ideally reproduced by a one-step imprinting process. In our experiments, the whole integration structure including $26 \mu \mathrm{m}$ deep microfluidic channels and about $90 \mu \mathrm{m}$ deep fiber channels were perfectly transferred from the glass master into PMMA by optimizing the imprinting conditions. (iii) PMMA replica masters originating from the same glass master have a better reproducibility than masters fabricated one-by-one using the conventional technique. In addition, PMMA replica masters are superior to masters made from glass, silicon, and photoresist materials in regard to their reusability. If desired, PMMA replica masters can be restructured with a new design through imprinting.

In summary, the ease of replicating multiple PMMA masters from the same original glass master, and the efficiency and low costs of the whole process provide a powerful platform for the massive production of PDMSbased disposable devices. Furthermore, other compatible polymers, such as polycarbonate sheets that allow for high glass temperature $\left(150^{\circ} \mathrm{C}\right)$, may be developed and applied as materials for replica masters for massive production of the PDMS-based disposable devices.

The authors gratefully acknowledge financial support of the National Science Fund for Distinguished Youth Scholars of China (No. 20125514), National Natural Science Foundation of China (No. 20437020, 50273046), and the Major Research Program of Chinese Academy of Sciences (KZCX3-SW-432).

Received November 27, 2004

\section{References}

[1] Neuhoff, V., Electrophoresis 2000, 21, 3-11.

[2] Seiler, K., Harrison, D. J., Manz, A., Anal. Chem. 1993, 65, 1481-1488.

[3] Jacobson, S. C., Culbertson, C. T., Daler, J. E., Ramsey, J. M., Anal. Chem. 1998, 70, 3476-3480.

[4] Harrison, D. J., Manz, A., Fan, Z., Luedi, H., Widmer, M. H., Anal. Chem. 1992, 64, 1926-1932.

[5] Manz, A., Miyahara, Y., Miura, J., Watanabe, Y., Miyagi, H., Sato, K., Sens. Actuators B 1990, 1, 249-255.

[6] Su, R.-G., Lin, J.-M., Qu, F., Yamada, M., Anal. Chim. Acta 2004, 508, 11-15.

[7] Becker, H., Gärtner, C., Electrophoresis 2000, 21, 12-26.

[8] Effenhauser, C. S., Bruin, G. J. M., Paulus, A., Ehrat, M., Anal. Chem. 1997, 69, 3451-3457.
[9] Duffy, D. C., McDonald, J. C., Schueller, O. J. A., Whitesides, G. M., Anal. Chem. 1998, 70, 4974-4984.

[10] McDonald, J. C., Duffy, D. C., Anderson, J. R., Chiu, D. T., Wu, H., Schueller, O. J. A., Whitesides, G. M., Electrophoresis 2000, 21, 27-40.

[11] Ng, J. M. K., Gitlin, I., Stroock, A. D., Whitesides, G. M., Eletrophoresis 2002, 23, 3461-3473.

[12] McCormick, R. M., Nelson, R. J., AlonsoAmigo, M. G., Benvegnu, J., Hooper, H. H., Anal. Chem. 1997, 69, 26262630.

[13] Wang, J., Pumera, M., Chatrathi, M. P., Escarpa, A., Konrad, R., Griebel, A., Dorner, W., Lowe, H., Electrophoresis 2002, 23, 596-601.

[14] Chen, Z.-F., Gao, Y.-H., Su, R.-G., Li, C.-W., Lin, J.-M., Electrophoresis 2003, 24, 3246-3252.

[15] Chen, Z.-F., Gao, Y.-H., Lin, J.-M., Su, R.-G., Xie, Y., J. Chromatogr. A 2004, 1038, 239-245.

[16] Olsen, K. G., Ross, D. J., Tarlov, M. J., Anal. Chem. 2002, 74, 1436-1441.

[17] Johnson, T. J., Ross, D., Locascio, L. E., Anal. Chem. 2002, $74,45-51$.

[18] Roberts, M. A., Rossier, J. S., Bercier, P., Girault, H., Anal. Chem. 1997, 69, 2035-2042.

[19] Locascio, L. E., Perso, C. E., Lee, C. S., J. Chromatogr. A 1999, 857, 275-284.

[20] McDonald, J. C., Whitesides, G. M., Acc. Chem. Res. 2002, 35, 491-499.

[21] Delamarche, E., Bernard, A., Schmid, H., Michel, B., Biebuyck, H., Science 1997, 276, 779-781.

[22] Auroux, P. A., lossifidis, D., Reyes, D. R., Manz, A., Anal. Chem. 2002, 74, 2637-2652.

[23] Duffy, D. C., Gillis, H. L., Lin, J., Sheppard, N. F., Kellogg, G. J., Anal. Chem. 1999, 71, 4669-4678.

[24] McDonald, J. C., Chabinyc, M. L., Metallo, S. J., Anderson, J. R., Stroock, A. D., Whitesides, G. M., Anal. Chem. 2002, 74, 1537-1545.

[25] Khoury, C., Mensing, G. A., Beebe, D. J., Lab Chip 2002, 2, 50-55.

[26] Sudarsan, A. P., Ugaz, V. M., Anal. Chem. 2004, 76, 32293235.

[27] Zhao, D. S., Roy, B., McCormick, M. T., Kuhr, W. G., Brazill, S. A., Lab Chip 2003, 3, 93-99.

[28] Martynova, L., Locascio, L. E., Gaitan, M., Kramer, G. W., Christensen, R. G., MacCrehan, W. A., Anal. Chem. 1997, $69,4783-4789$

[29] Liu, Y., Fanguy, J. C., Bledsoe, J. M., Henry, C. S., Anal. Chem. 2000, 72, 5939-5944.

[30] Xiong, S.-X., Han, H.-W., Zhao, R., Chen, Y., Liu, G.-Q., Biomed. Chromatogr. 2001, 15, 83-88.

[31] Li, H.-F., Lin, J.-M., Su, R.-G., Uchiyama, K., Hobo, T., Electrophoresis 2004, 25, 1907-1915.

[32] Odian, G., Principles of Polymerization, John Wiley \& Sons, New York 1991.

[33] Morra, M., Occhiello, E., Marola, R., Garbassi, F., Humphrey, P., Johnson, D., J. Colloid Interface Sci. 1990, 137, 11-24.

[34] Wang, S.-C., Perso, C. E., Morris, M. D., Anal. Chem. 2000, 72, 1704-1706.

[35] Xu, W., Uchiyama, K., Shimosaka, T., Hobo, T., J. Chromatogr. A 2001, 907, 279-289. 